\title{
Effects of Port Congestion in the Gate Control List Scheduling of Time Sensitive Networks
}

Kentis, Angelos Mimidis; Berger, Michael Stübert; Soler, José

Published in:

Proceedings of 8th International Conference on the Network of the Future (NOF)

Link to article, DOI:

10.1109/NOF.2017.8251236

Publication date:

2017

Document Version

Publisher's PDF, also known as Version of record

Link back to DTU Orbit

Citation (APA):

Kentis, A. M., Berger, M. S., \& Soler, J. (2017). Effects of Port Congestion in the Gate Control List Scheduling of Time Sensitive Networks. In Proceedings of 8th International Conference on the Network of the Future (NOF) (pp. 138-140). IEEE. https://doi.org/10.1109/NOF.2017.8251236

\section{General rights}

Copyright and moral rights for the publications made accessible in the public portal are retained by the authors and/or other copyright owners and it is a condition of accessing publications that users recognise and abide by the legal requirements associated with these rights.

- Users may download and print one copy of any publication from the public portal for the purpose of private study or research.

- You may not further distribute the material or use it for any profit-making activity or commercial gain

- You may freely distribute the URL identifying the publication in the public portal 


\title{
Effects of Port Congestion in the Gate Control List Scheduling of Time Sensitive Networks
}

\author{
Angelos Mimidis Kentis, Michael Stubert Berger and Jose Soler \\ Technical University Of Denmark, Department of Photonics Engineering \\ Lyngby, Denmark \\ \{agmimi, msbe,joss\}@fotonik.dtu.dk
}

\begin{abstract}
Time Sensitive Networking (TSN) can provide deterministic traffic behavior over Ethernet networks, for time sensitive traffic, whilst also bound the delay/jitter. To do so, the IEEE TSN working group introduced a network-wide transmission port scheduling mechanism. The duration of this schedule is directly related with the delay; hence reducing it can be beneficial within the TSN paradigm. This paper investigates the effects of port congestion, in the duration of the network wide schedule. A congested port can make scheduling more complex, leading to longer network-wide schedules. To verify this, the same set of experiments was repeated, with and without considering port congestion during path allocation. The computed paths were given as input to an implementation of the shifting bottleneck heuristic algorithm. The shifting bottleneck heuristic, computed the network-wide gating schedule. The results show that with port congestion as a metric during path allocation the duration of the gating schedule in multipath networks can be reduced up to $26 \%$.
\end{abstract}

Keywords—TSN; Traffic Scheduling;

\section{INTRODUCTION}

Traffic in Time Sensitive Networks (TSN) has very stringent delay/jitter requirements and requires deterministic behavior from the network. In order to achieve these requirements the IEEE TSN working group [1] proposed a mechanism that can schedule Time Sensitive (TS) traffic over the network, whilst also bound the end-to-end delay/jitter. In this mechanism, every transmitting port in the network has a set of gates that control which queue can transmit at any given time. By scheduling these gates, isolating TS and Best Effort (BE) traffic into different queues and synchronizing the network-wide gating schedules, the requirements of TS traffic can be met.

In TSN, path allocation is based on shortest path calculations, using a variety of metrics (e.g. delay, bandwidth). Port congestion is also considered, but defined as optional. This paper argues that port congestion can severely affect the duration of the network-wide gating schedule. This is very important, considering that the duration of the schedule is directly related to the average end-to-end delay of TS flows. To evaluate the effect of port congestion, this paper presents a comparison, in terms of schedule duration, between scenarios with and without considering port congestion during path allocations. To do so a path allocation algorithm was developed based on K-Shortest path calculations. However, this algorithm, uses port congestion, modeled as number of flows per transmitting port, as a metric. The results show that in multipath networks, considering port congestion can shorten the duration of the gating schedule (up to $26 \%$ ).

\section{BACKGROUND AND RELATED WORK}

\section{A. Time Sensitive Networks}

For most traffic types (e.g. video streaming, file transferring), the Best Effort (BE) treatment is sufficient. This is because these traffic types are of low criticality, and can accommodate for peaks and fluctuations in delay/jitter (e.g. with the use of buffering, for streaming). However certain types of traffic, like signals for industrial automation and automotive environments have a high mission criticality. This implies that the effects of delay/jitter can have a substantial effect in service quality (e.g. steering controls for automotive environments must not be delayed due to network congestion). The approach so far, has been to develop, install and maintain separate network infrastructures for mission-critical TS traffic. These networks provide both deterministic traffic behavior and bounded delay/jitter. However this approach has two drawbacks. (1) It has led to a number of different network architectures, each tailored for only a specific environment (2) Most of these environments require the deployment of an Ethernet network, to carry BE traffic. This implies the existence of two separate networks, increasing the network complexity and associated costs. To address this, the IEEE TSN working group proposed a set of standards that extent IEEE 802.1. These standards define an Ethernet based, architecture that provides determinism for TS traffic, whilst also allow BE traffic to share the same infrastructure. Determinism is provided by means of traffic scheduling and packet preemption. For the scope of this paper only traffic scheduling is of interest. To ensure that BE traffic will not influence TS traffic; the IEEE TSN working group introduced a gating mechanism on each of the transmitting ports of the network. This mechanism controls which queue can transmit over the port at any given time Fig. 1 illustrates an example of this architecture. There traffic is divided into two queues by means of a traffic classifier. Queue A holds TS traffic and Queue B holds BE traffic. A local Gate Control List (GCL) holds the states of the queues (blocked or unblocked) with respect to time. The logic of this architecture is the following. Schedule the GCLs of the network, so that when a TS queue has frames to transmit, then it should be in the unblocked 
state. Else block the TS queue and unblock the BE queue. This way, the BE traffic will not interfere with the TS traffic and the TS traffic will be prioritized over the BE traffic.

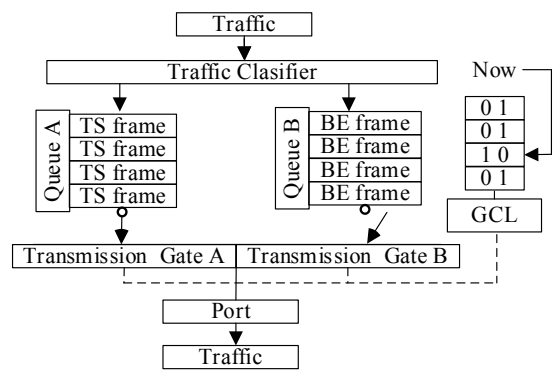

Fig. 1. Traffic Scheduling

\section{B. Related work}

In [2], the author proposes an algorithm that performs ratebased path selection in access networks. However, instead of directly monitoring the bandwidth utilization, the average input rate of TCP flows is used instead. The assumption is that paths with a low average rate are more likely to be congested. In [3], the authors also point that using the shortest path can lead to network congestion. They propose a tie-breaking algorithm, when more than one shortest paths exists, based on historical link-load data. The authors of [4] propose a path selection algorithm that assigns probabilities to the equal cost trees from which the final path will be selected. The probabilities are based on link-load estimations. With regards to routing of TS flows, [5] proposes a heuristic methodology for computing the TS flows paths. A set of algorithms is proposed, that find edge-disjoint paths for each TS flow. This approach yields good results with regards to the delay/jitter requirements of the TS flows. However, it does not scale for big number of TS flows. Finally, [6] proposes a heuristic methodology for routing Audio Video Bridging (AVB) streams, in TSN. The algorithm takes as input the network topology, together with the already allocated TS flows and attempts to allocate the AVB streams. While congestion aware path allocation and routing of TS flows in TSN have been researched, the effect of port congestion in the duration of the GCLs' schedules has not.

\section{SCHEDUling OF THE GATE CONTROL LISTS}

As stated in section II.A, supporting traffic scheduling within a TSN network requires populating the GCLs. Due to, the size of modern networks, the complex traffic patterns and the need for synchronization amongst the GCLs, generating these schedules is computationally intensive. Finding optimal schedules (i.e. schedules that minimize the delay of TS flows) would require exceedingly long computational runtimes, hence the schedules of the GCLs are generated offline, with the use of heuristics. Heuristics return approximate (nonoptimal) solutions to the given problem. The quality of the solution depends on many variables, like the performance of the algorithm and the available runtime. As stated in Section I, for the GCL scheduling problem the quality of a solution is related to the duration of the computed schedule.

\section{Path Allocation in Time Sensitive Networks}

Path allocation in TSN is done using shortest path bridging by means of the Intermediate System - Intermediate System protocol (IS-IS). There, paths are selected by defining explicit trees between communicating hosts. Multiple metrics are available for selecting the best tree, like link bandwidth and delay. In the case of more than one shortest path, this scheme can break ties based on how congested each path is in terms of available bandwidth. However this functionality is defined as optional. This paper argues on the importance of monitoring port congestion and regarding it as a metric. In addition to causing port congestion, assigning flows over the shortest path does not take advantage of the offline nature of scheduling in TSN which allows for more complex schemes.

\section{PROTOTYPE IMPLEMENTATION}

To monitor the effects of port congestion in the scheduling of the GCLs, a path allocation algorithm was developed. The algorithm is based on K-shortest path calculations, but also takes into account port congestion (modeled based on the number of TS flows that the port is serving). The algorithm takes as input the following: (1) A set of network nodes: This includes all the forwarding elements of the network. (2) A set of TSN talkers: Talkers in the TSN architecture initiate TS flows. (3) A set of TSN listeners: Listeners in the TSN architecture receive TS flows. (4) $\underline{A}$ set of links: This includes both node-to-node links and node-to-talker/listener links. (5) A set of link annotations: The annotations define the cost metric associated with a link. (6) A set of TS flows: Defined as a source/destination pair.

Initially no TS flow is scheduled on the network (all ports serve zero TS flows). Then the algorithm starts scheduling the TS flows one by one. Since each TS flow is defined as a source/destination pair, the algorithm performs a K-Shortest path computation for this pair. The reasons for performing the K-Shortest computations are: (1) It limits the search space for selecting the final path. (2) It can limit the accumulated metric of a path, by varying how many paths the K-Shortest path algorithm returns. Then the algorithm ranks each path, returned from the K-Shortest path algorithm, based on an evaluation function. This function evaluates the effect of each path, by temporarily adding it to the current active path schedule, and then monitors the overall state of port congestion. Since the goal is to minimize port congestion, the evaluation is based on (1), where $p_{i}$ is the number of flows port $i$ is serving, $N$ is the total number of transmitting ports in the network and $\mu$ is the current mean value of served flows per port.

$$
s t d=\sqrt{\frac{\sum_{1}^{\mathrm{N}}\left(p_{i}-\mu\right)^{2}}{\mathrm{~N}}}
$$

After evaluating all paths, the best (the one of lowest value) is added to the active path schedule. When all flows are scheduled, the algorithm terminates. In Fig. 2, (1) $\underline{F}$ : Represents the TS flows that need to be scheduled. (2) $\underline{S}$ : Holds the path allocated to each flow. On each loop an additional path is added to $S$. (3) $\underline{P}$ : Holds the paths returned by the K-Shortest path algorithm for a specific TS flow. (4) $\underline{K}$ - 
shortestPath $(f)$ : Returns a set of $\mathrm{k}$ paths that can serve TS flow $f$. (5) eval(S,p): Evaluates the effect of assigning path $p$ to current solution $S$. (6) $\underline{\text { best }(P)}$ : Returns the best out of $P$. (7) $\underline{n \operatorname{ext}(F)}$ : Returns the next element of $F$.

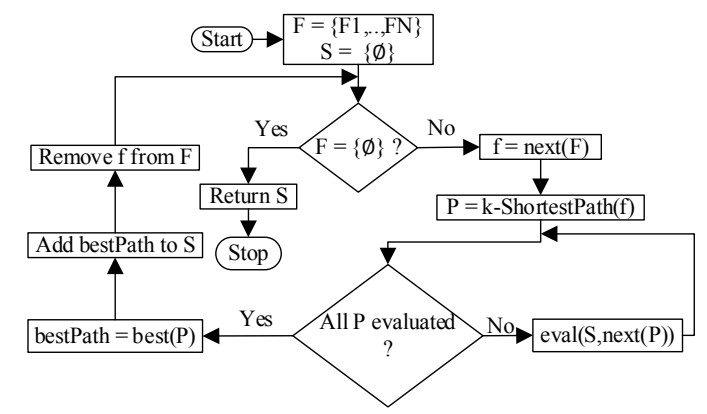

Fig. 2. The congestion-aware, path selection algorithm.

The algorithm was implemented as an application in an open source SDN controller. This approach simplified the development process, as it reused some core functionalities present in the SDN controller (e.g. topology and path discovery and network state overview). The topologies were generated using Mininet. The SDN controller and Mininet were used as tools to generate the paths and were not intended to simulate a live network scenario.

The paths provided by the path allocation algorithm, were given as input to an implementation of the shifting bottleneck heuristic which calculated the network-wide GCL schedules. These schedules were then evaluated based on their overall duration (shorter is better).

\section{RESULTS}

Two network topologies were used. (1) A ring topology with two links per node pair and (2) a partial-mesh topology. In both topologies 10 Talkers initiated a number of bidirectional traffic flows to 3 Listeners. These topologies were selected because: (1) They are commonly deployed and (2) they have an inherently different behavior when it comes to multi-path routing. Mesh topologies have multiple paths between any two hosts while ring topologies are more restricted. The experiments also varied in the number of flows each talker initiated to each listener. This way the algorithm was also evaluated with respect to how it scales.

The results are presented in TABLE I, the first five columns describe the experiment itself (From left to right: topology type, \#talkers, \#listeners, number of bidirectional flows initiated per talker/listener pair and finally the number of shortest paths returned by the K-Shortest Path algorithm). The last three columns present the results (From left to right: schedule duration with the proposed algorithm, schedule duration with only shortest path and finally the difference as a percentage.

As the results show, the effects of using port congestion as a metric depend on the topology and network state. In the ringbased experiments the results between the two path-allocation schemes, were almost identical. This can be contributed to the limited amount of paths between any two listener/talker pairs. In contrast, the experiments conducted over the mesh topology showed considerable reduction (up to $26 \%$ ) in the duration of the network-wide gating schedule when port congestion was used as a metric. This again can be contributed to the fact that mesh topologies have many paths between any two talker/listener pairs.

Every experiment presented in this section yielded identical results on each repetition. So the number of repetitions per experiment was limited to 3 .

TABLE I. Schedule duration with/without considering port congestion

\begin{tabular}{|l|l|l|l|l|l|l|l|}
\hline Topo & \#T & $\# \mathbf{L}$ & $\mathbf{\# F}$ & $\mathbf{\# P}$ & Cong. aware & Non cong. aware & Gain \% \\
\hline Ring & 10 & 3 & 2 & 2 & 325 & 320 & -1.56 \\
\hline Ring & 10 & 3 & 3 & 2 & 475 & 480 & 1.04 \\
\hline Ring & 10 & 3 & 4 & 2 & 625 & 625 & 0 \\
\hline Mesh & 10 & 3 & 2 & 4 & 175 & 210 & 16.67 \\
\hline Mesh & 10 & 3 & 3 & 4 & 245 & 320 & 23.44 \\
\hline Mesh & 10 & 3 & 4 & 4 & 325 & 440 & 26.17 \\
\hline
\end{tabular}

\section{CONCULSION}

This paper evaluated the effects of port-congestion in the duration of the network-wide GCL schedule in the TSN paradigm. In order to do so a, congestion aware, path allocation algorithm was developed and evaluated on different scenarios. Finally the paths computed by the algorithm were given as input to a heuristic solver, which calculated the network-wide schedules for the GCLs. The results indicate that considering port congestion, in networks with multiple available paths, can result in substantially shorter GCL schedules (up to $26 \%$ ). Given the results and the importance of low delay communication for TS flows, port congestion can be considered as an important metric for the path allocation process in the TSN paradigm.

\section{ACKNOWLEDGMENT}

This work receives support from the "Intelligent $5 \mathrm{G}$ mobile Ethernet Radio Access Network (ERAN)" project founded by Innovation Fund Denmark.

\section{REFERENCES}

[1] IEEE TSN Working Group, Available: http://www.ieee802.org/1/pages/tsn.html. [Accessed: 20-Apr-2017].

[2] Y. Nakayama, "Rate-Based Path Selection for Shortest Path Bridging in Access Networks," IEEE ICC 2014 - Communication QoS, Reliability and Modeling Symposium, pp. 1266-1271, 2014.

[3] D. Allan, J. Farkas, and S. Mansfield, "Intelligent Load Balancing for Shortest Path Bridging", 164 IEEE Communications Magazine, July 2012, pp. 163-167, 2012.

[4] Y. Nakayama, "Optimization of ECT selection probability in SPBM networks,", IEICE Communications Express, Vol.3, No.1, pp. 33-38 vol. $3,2014$.

[5] N. G. Nayak, D. Frank, and K. Rothermel, "Software-defined Environment for Reconfigurable Manufacturing Systems", Proceedings of the 5th International Conference on Internet of Things, 2015.

[6] S. M. Laursen, P. Pop, and K. Lyngby, "Routing Optimization of AVB Streams in TSN Networks," ACM SIGBED Review, 13(4), pp. 43-48, 2016.

[7] J. Adams, E. Balas, and D. Zawack, "The Shifting Bottleneck Procedure for Job Shop Scheduling," Manage. Sci., vol. 34, no. 3, pp. 391-401, 1988. 